\title{
Transforaminal versus Intra-Articular Facet Corticosteroid Injections for the Treatment of Cervical Radiculopathy: A Randomized, Double-Blind, Controlled Study
}

\author{
N.J. Bureau, T. Moser, J.H. Dagher, D. Shedid, M. Li, P. Brassard, and B.E. Leduc
}

A O OEBM

\begin{abstract}
BACKGROUND AND PURPOSE: Transforaminal corticosteroid injections can be performed in the management of cervical radiculopathy but carry the risk of catastrophic complications. This study compares the efficacy of transforaminal and facet corticosteroid injections at 4 weeks' follow-up.

MATERIALS AND METHODS: We randomly assigned 56 subjects to receive CT-guided transforaminal (15 men, 13 women; mean age, 52 years; range, 28-72 years) or facet ( 8 men, 20 women; mean, 44 years; range, 26-60 years) injections. The primary outcome was pain severity rated on a Visual Analog Scale (0-100). Secondary outcomes were the Neck Disability Index and the Medication Quantitative Scale.

RESULTS: In the intention-to-treat and as-treated analyses, for a mean baseline score, facet injections demonstrated a significant pain score reduction of $45.3 \%(95 \% \mathrm{Cl}, 21.4-69.2)$ and $37.0 \%(95 \% \mathrm{Cl}, 9.2-64.7)$, while transforaminal injections showed a nonsignificant pain score reduction of $9.8 \%(95 \% \mathrm{Cl},+11.5-31.2)$ and $17.8 \%(95 \% \mathrm{Cl},+6.6-42.2)$. While facet injections demonstrated an improvement in the Neck Disability Index score of $24.3 \%(95 \% \mathrm{Cl},+2.9-51.5)$ and $20.7 \%(95 \% \mathrm{Cl},+6.2-47.6)$ as opposed to transforaminal injections of $9.6 \%$ $(95 \% \mathrm{Cl},+15.2-34.4)$ and $12.8 \%(95 \% \mathrm{Cl},+11.2-36.7)$, the results were not statistically significant. Noninferiority of facet to transforaminal injections was demonstrated for baseline pain scores of $\leq 60$, while noninferiority analysis was inconclusive for baseline pain scores of $\geq 80$ and for the Neck Disability Index. Neither intervention showed a significant medication-intake score reduction with time.
\end{abstract}

CONCLUSIONS: Facet injections are effective for the treatment of cervical radiculopathy and represent a valid and safer alternative to transforaminal injections.

ABBREVIATIONS: IFSI = intra-articular facet corticosteroid injection; NDI = Neck Disability Index; MQS = Medication Quantitative Scale; TFSI = transforaminal corticosteroid injection; VAS = Visual Analog Scale

C ervical radiculopathy is a debilitating condition caused by the irritation of a cervical spinal nerve root. Patients typically present with pain radiating to the upper arm and a combination of sensory disorder, altered reflexes, or motor weakness. ${ }^{1}$ It affects approximately 1 person per 1000 of population per year and is most often caused by degenerative spondylosis and/or a disk her-

Received April 16, 2014; accepted after revision May 12

From the Department of Radiology (N.J.B., T.M.), Research Center (N.J.B., T.M.), Department of Surgery (D.S.), Division of Neurosurgery, and Department of Medicine (B.E.L.), Centre hospitalier de l'Université de Montréal, Montreal, Quebec, Canada; Institut de réadaptation Gingras-Lindsay-de-Montréal (J.H.D.), Université de Montréal, Montreal, Quebec, Canada; Department of Surgery (M.L.), Division of Neurosurgery, Hôpital Maisonneuve-Rosemont, Université de Montréal, Montreal, Quebec, Canada; and Division of Clinical Epidemiology (P.B.), McGill University Health Center, Montreal, Quebec, Canada.

This work was funded by the Fonds de recherche du Québec-Santé (Quebec Government Funding Agency) (grant 21230-2)

Paper previously presented at: Annual Meeting of the American Society of Neuroradiology and the Foundation of the ASNR Symposium, May 17-22, 2014; Montreal, Quebec, Canada. niation. ${ }^{2}$ Historically, approximately $30 \%$ of patients have required surgery. ${ }^{3}$ Most patients will be treated medically, including rest, analgesics, nonsteroidal anti-inflammatory drugs, physical therapy, and corticosteroid injections.

Disk herniation and degenerative changes occurring at the intervertebral disk level, the uncovertebral joint, and/or the facet joint can potentially irritate the spinal nerve root by 2 major mechanisms: production of mediators of inflammation ${ }^{4}$ and compression of the nerve. The presumed therapeutic effect of corticosteroid injections is the suppression of the inflammatory cascade. Transforaminal corticosteroid injections (TFSI) allow delivery of a high concentration of corticosteroids directly and

\footnotetext{
Please address correspondence to Nathalie J Bureau, MD, Department of Radiology, Centre hospitalier de l'Université de Montréal, 1058 St-Denis, Montreal, Quebec, Canada, H2X 3J4; e-mail: nathalie.bureau@umontreal.ca

- Indicates open access to non-subscribers at www.ajnr.org

Evidence-Based Medicine Level 1.

http://dx.doi.org/10.3174/ajnr.A4026
}

AJNR Am J Neuroradiol 35:1467-74 Aug 2014 www.ajnr.org 
precisely at the site of the involved spinal nerve and are used to treat cervical radiculopathy. ${ }^{5,6}$ In the past 15 years, a significant number of catastrophic neurologic complications after TFSI have been reported in the literature. ${ }^{7}$ Although the exact prevalence of these devastating adverse events is unknown, some authors have questioned the continued use of TFSI, ${ }^{8}$ while others advocate technical strategies to improve the safety of the procedure ${ }^{9,10}$ or alternative approaches, which potentially carry fewer risks. ${ }^{8,11}$

Preliminary work by Kim et al in $2005^{12}$ and by Richarme et al in $2008^{11}$ suggested that intra-articular facet steroid injections (IFSIs) could be effective in patients with cervical radiculopathy secondary to disk herniation. Anatomically, the facet joint ventral recess is in close proximity to the spinal nerve root. Furthermore, Richarme et $\mathrm{al}^{11}$ suggested that leakage of contrast into the foramen could be a potential mechanism of action. Therefore, using a facet joint injection approach to deliver corticosteroids in the vicinity of the injured spinal nerve root appears to be a viable alternative to the riskier transforaminal approach.

We hypothesized that IFSI could be at least as effective as TFSI for the treatment of cervical radiculopathy, and we devised this study to compare, at 4 weeks' follow-up, the efficacy of IFSI with TFSI in subjects with cervical radiculopathy of at least 1 month's duration due to degenerative spondylosis and/or a disk herniation.

\section{MATERIALS AND METHODS Subjects}

Recruitment. The Research Ethics Committee of our institution approved this study. All subjects received written and verbal information and gave their written consent. The study was performed according to the Declaration of Helsinki, was approved by the scientific committee of the Research Funding Agency of the Quebec Government (grant 21230-2), and was registered at the University of Montreal as part of a master's thesis in biomedical science.

Prospectively, 56 adults with cervical radiculopathy, capable of giving written consent, were enrolled in the study. The subjects were recruited from the hospital community. One of 2 physiatrists or 1 of 2 neurosurgeons performed a clinical evaluation of the subjects to confirm the diagnosis of cervical radiculopathy according to eligibility criteria, similar to criteria used in previous studies, ${ }^{5,9}$ and to identify the presence of exclusion criteria.

The eligibility criteria were the following: evidence of a cervical radiculopathy involving 1 spinal nerve of at least 1 month's duration refractory to medical treatment; symptoms of cervical pain radiating to the upper limb; and signs of altered sensations, abnormal reflexes, or motor weakness caused by degenerative spondylosis and/or disk herniation as documented at CT or MR imaging and a current mean pain score of $\geq 6$ on a Verbal Analog Scale of 0 (no pain) to 10 (worse pain imaginable). The exclusion criteria were the following: evidence of vertebral fracture, tumor, or infection of the cervical spine; treatment with cervical corticosteroid injections within the past 3 months; coagulopathy; and allergy to iodinated contrast media. One of 2 radiologists reviewed the imaging studies to confirm the presence of degenerative spondylosis and/or a disk herniation at the level of the involved spinal nerve and to exclude other pathology.
Enrollment in the Study. The research assistant performed a telephone interview with the subjects who agreed to participate in the study, to discuss the research protocol in detail, to collect medical and demographic data, and to schedule an appointment for the intervention within 2 weeks of their clinical evaluation. The subjects were informed of the risk of minor adverse effects and of major complications after the procedure. On request, the subjects could meet with one of the researchers for any inquiry concerning their participation in the study.

Randomization. Patients were randomized to 1 of 2 groups: treatment with TFSI at the level of the involved spinal nerve root or treatment with IFSI of the facet joint adjacent to the involved spinal nerve root. The randomization sequence with block sizes of 8 was computer-generated by a person not otherwise directly involved with the subjects. The envelopes were sealed and sequentially numbered from 1 to 56 . The randomization was done without stratification to allow evaluating the demographic and clinical aspects of interest. On the day of the procedure, the research assistant gave the sealed envelope matching the sequential number of the subject to the radiologist in charge of performing the intervention. The radiologist checked the content of the envelope and resealed the envelope before returning it to the assistant. Hence, the assistant remained blinded to the type of injection for the duration of the study.

\section{Interventions}

One of 2 musculoskeletal radiologists with 10 and 18 years of experience in interventional spine procedures performed the injections. The TFSIs were performed according to a standard technique, ${ }^{9}$ and the IFSIs were performed by using a lateral approach. The setup of the patient, the sequential steps of the technique, and the material used were identical, with the exception of the targeted site of injection, which was the posterior and lateral aspects of the neural foramen for the TFSI (Fig $1 A$ ) and the facet joint space for the IFSI (Fig 1B). This method ensured that the subjects remained blinded to the type of injection they received.

The subject was placed in the supine position, with his or her head turned $45^{\circ}$ to the side contralateral to the injection. Scout images of the targeted neural foramen were obtained. The appropriate entry site was marked on the skin. Then, the skin was prepped and draped in the usual fashion. The skin and subcutaneous tissue were anesthetized with lidocaine $1 \%$. Then, a $22-$ ga, 2.5-inch spinal needle was advanced by using intermittent CT fluoroscopy (Brilliance-64; Philips Healthcare, Best, the Netherlands) with a collimation of $2.5 \mathrm{~mm} \times 4$ images set. Once the needle was in the appropriate location, $0.5-1.0 \mathrm{~mL}$ of contrast material (iohexol, Omnipaque 240; GE Healthcare Canada, Mississauga, Canada) was injected by using minibore tubing connected to a 3-mL syringe; and at the end of the injection, CT fluoroscopy images were acquired by the radiologist to exclude an intravascular position of the needle and to confirm adequate distribution of the contrast material. Then, 1 $\mathrm{mL}$ of dexamethasone sodium phosphate, $10 \mathrm{mg} / \mathrm{mL}$ was injected by using a $1-\mathrm{mL}$ syringe. Then, the needle was withdrawn and the subject was observed for 30 minutes. 

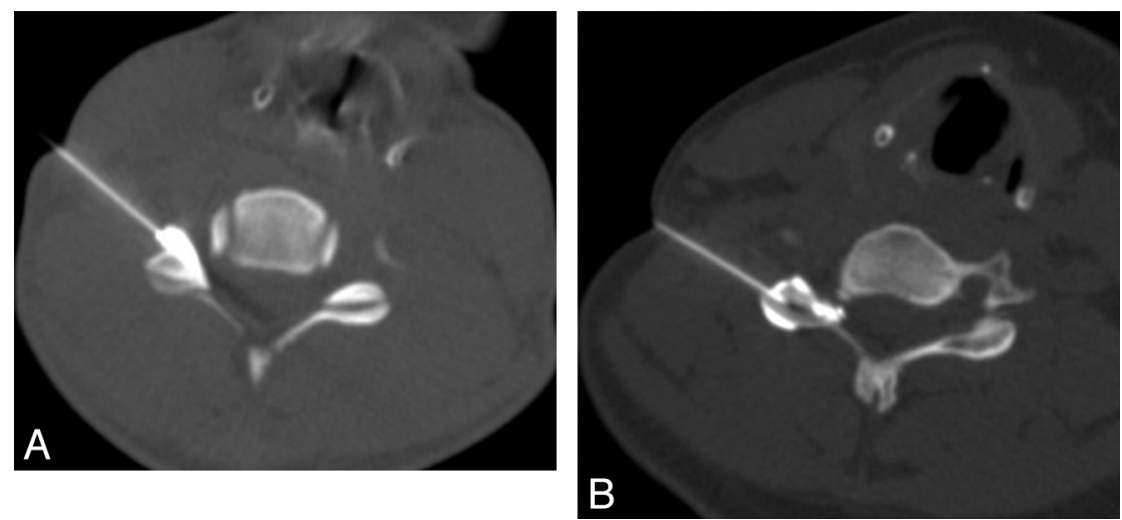

FIG 1. A, CT-guided transforaminal corticosteroid injection. The needle is positioned in the posterolateral aspect of the foramen with contrast media flowing in the foramen. $B, C T$-guided intra-articular facet corticosteroids injection. The needle is positioned in the facet joint. Contrast media injection confirms an intracapsular distribution.

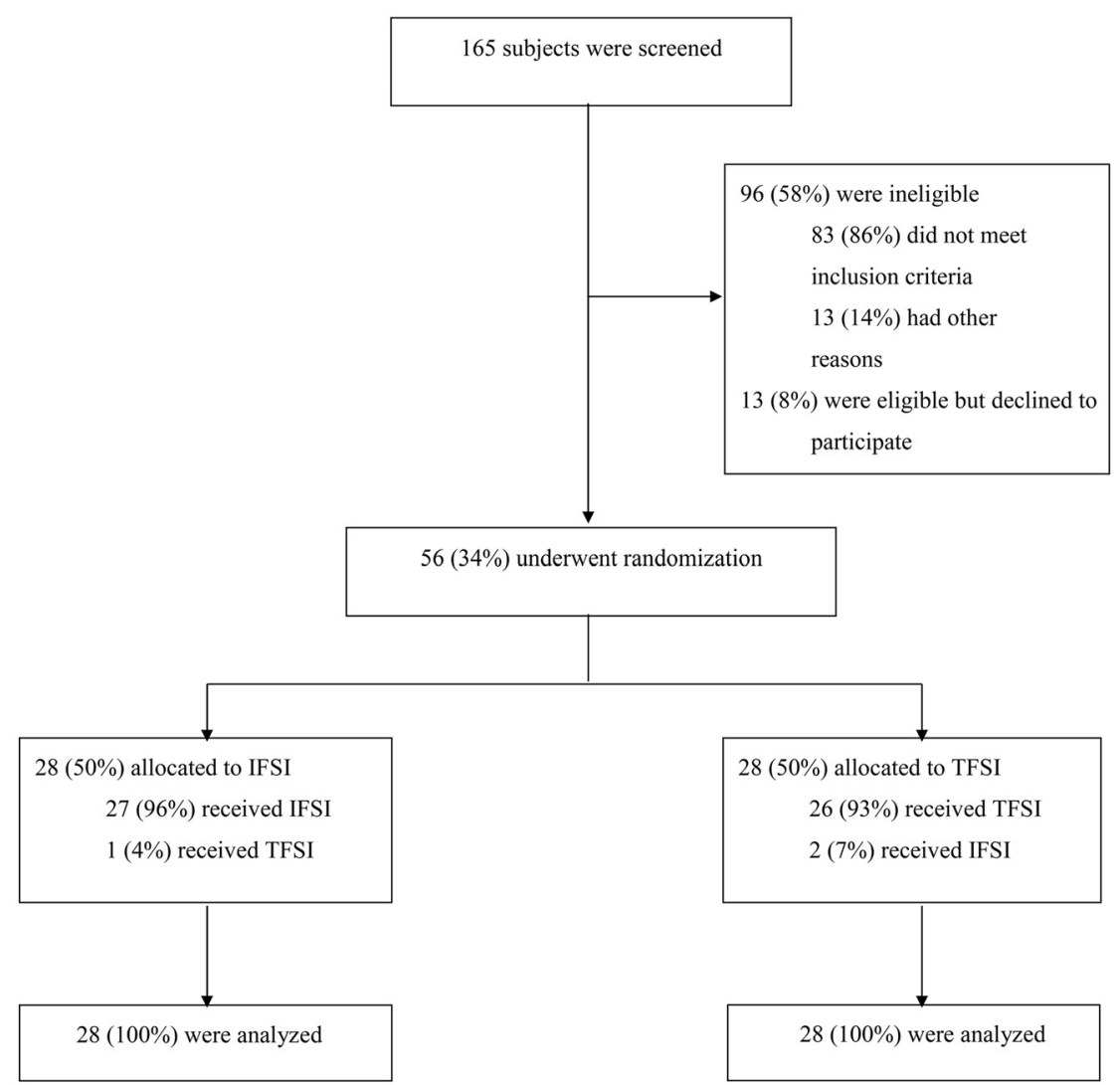

FIG 2. Flow diagram of the progress of subjects through the phases of the study.

\section{Outcome Measures}

The primary outcome was pain severity rated on a Visual Analog Scale (VAS) measured 4 weeks after the intervention. Subjects were asked to indicate the mean pain level experienced during the past few days on a VAS consisting of a straight line extending from 0 (no pain) to $100 \mathrm{~mm}$ (worse pain imaginable). Secondary outcome measures were the Neck Disability Index (NDI) ${ }^{13}$ and the Medication Quantitative Scale (MQS). ${ }^{14}$ The NDI is a valid questionnaire, which measures the impact of neck pain on everyday life activities related mainly to personal care, work, sleeping, driving, reading, and recreation. It comprises 10 items, each scored from 0 (no disability) to 5 (total disability). The minimal clinically important difference is $5(10 \%)$. We used the MQS to quantify medication use on a weekly basis after the intervention. Scores were calculated for each pain-related medication based on weights assigned by pharmacologic class and dosage level and were summed to yield the total MQS score. Although the lowest possible MQS score is 0 , there is no definite upper limit. The minimal clinically significant reduction in MQS score is 4 . At baseline, subjects were instructed to continue taking their usual medication after the procedure and to keep a written daily record of the type and dose of their medication intake during the next 4 weeks.

\section{Follow-Up Time Points}

On the day of the intervention, before randomization, the subjects met with the research assistant at the university hospital to sign the consent form and to complete the baseline VAS and the NDI questionnaire. Thirty minutes after the intervention, the assistant met with the subjects to assess any immediate adverse reactions. The subjects were asked to rate the worst pain experienced during the intervention and their level of pain at the current time on a Verbal Analog Scale $(0-10)$. The assistant met again with the subjects 4 weeks after their procedure to complete the postintervention VAS and the NDI questionnaire. The subjects were questioned about any delayed adverse effects following the procedure. The medication record notebooks were also collected.

\section{Statistical Analysis}

It was determined that a sample size of 56 subjects, divided equally into the 2 groups, would be required to detect an efficacy in VAS pain score reduction of at least $30 \%$ in both groups, with a power of $80 \%$ and a type I error of $5 \%$ in 2 paired Student $t$ tests. Descriptive statistics were used to characterize the 2 subject groups at baseline, and the Student $t$ and $\chi^{2}$ tests with a 2 -sided $\alpha$ level of .05 were used to compare the characteristics between the 2 groups.

The main analyses followed the intention-to-treat principle, analyzing all patients according to randomization. In addition, an as-treated analysis was performed according to the type of injection the subjects received. Relative differences between postintervention and baseline scores were calculated for the VAS pain score and the NDI. The clinical efficacy of the IFSI and TFSI, in terms of VAS pain score reduction and NDI improvement, was defined as 
Table 1: Baseline characteristics of subjects (per randomization) treated with either transforaminal or intra-articular facet corticosteroid injections

\begin{tabular}{lccc} 
& \multicolumn{2}{c}{ Groups } & \\
\cline { 2 - 3 } \multicolumn{1}{c}{ Variables } & IFSI & TFSI & P Value \\
\hline Subjects (No.) & 28 & 28 & \\
Sex (No.) (\%) & & & .059 \\
Male & $20(29)$ & $15(54)$ & \\
Female & $44 \pm 8.3(26-60)$ & $52 \pm 11.1(29-72)$ & .007 \\
Age (yr) (mean) (range) & $14 \pm 20(1-84)$ & $17 \pm 21(1-84)$ & .649 \\
Duration of pain (mo) (mean) (range) & & & .357 \\
Imaging findings (No.) (\%) & $12(43)$ & $7(25)$ & \\
Disk herniation & $14(50)$ & $20(71)$ & \\
Spondylosis & $2(7)$ & $1(4)$ & .566 \\
Spondylosis/disk herniation & & & \\
Level of injection (No.) (\%) & $1(4)$ & $0(0)$ & \\
$\quad$ C3-C4 & $1(4)$ & $3(11)$ & \\
C4-C5 & $16(57)$ & $15(53)$ & \\
C5-C6 & $10(35)$ & $10(36)$ & \\
C6-C7 & & & \\
Side of injection (No.) (\%) & $13(46)$ & $17(61)$ & \\
Right & $15(54)$ & $11(39)$ & \\
Left & $61 \pm 17(23-95)$ & $63 \pm 18(17-85)$ & .691 \\
VAS (0-100) (mean) (range) & $21 \pm 8(10-44)$ & $19 \pm 7(5-30)$ & .301 \\
NDI (0-50) (mean) (range) & & & .030 \\
Employment status (No.) (\%) & $12(42.9)$ & $16(57.1)$ & \\
Working & $1(3.6)$ & $4(14.3)$ & \\
Retired & $15(53.5)$ & $8(28.6)$ & \\
Not working & $10(35.71)$ & $4(14.29)$ & \\
$\quad$ On sick leave with insurance & $0(0.00)$ & $2(7.14)$ & \\
On sick leave without insurance & $4(14.29)$ & $0(0.00)$ & \\
$\quad$ Workers' compensation & $1(3.57)$ & $2(7.14)$ & \\
$\quad$ On welfare & & & \\
\hline
\end{tabular}

\section{RESULTS}

Of 165 subjects who were screened, 56 were enrolled in the study from December 1, 2010, through September 30, 2013. The study flow chart is shown in Fig 2.

Among the eligible subjects at screening $(n=69), 81 \%(n=56)$ agreed to participate in the study. No subjects were lost to follow-up. Among the subjects randomized to receive an IFSI, 1 subject received a TFSI by a mistake of the radiologist. Among the subjects randomized to receive a TFSI, 2 subjects received an IFSI. In 1 case, the anterior recess of the articular facet was entered inadvertently. In the other case, the radiologist tried unsuccessfully to perform a TFSI at the C6-C7 level and finally opted to perform an IFSI instead.

Subject characteristics at baseline are presented in Table 1. The baseline characteristics of the 2 groups were similar except for age, sex, and employment status variables, for which a significant or almost-significant difference was found. In addition, those receiving TFSI had an apparent higher percentage with spondylosis $(71 \%)$ compared with disk herniation

Table 2: Pain severity and Neck Disability Index scores for intraarticular facet and transforaminal corticosteroid injections

\begin{tabular}{lcc}
\hline & \multicolumn{2}{c}{ Groups } \\
\cline { 2 - 3 } Variable & IFSI & TFSI \\
\hline Subjects (No.) & 28 & 28 \\
It & 29 & 27 \\
At & & \\
VAS\% (mean) (95\% CI) & $45.3(21.4-69.2)$ & $9.8(+11.5-31.2)$ \\
It & $37.0(9.2-64.7)$ & $17.8(+6.6-42.2)$ \\
At & & \\
NDI\% (mean) (95\% Cl) & $24.3(+2.9-51.5)$ & $9.6(+15.2-34.4)$ \\
It & $20.7(+6.2-47.6)$ & $12.8(+11.2-36.7)$ \\
At
\end{tabular}

Note:-VAS\% indicates the relative difference between postintervention and baseline VAS pain scores; NDI\%, the relative difference between postintervention and baseline Neck Disability Index scores; It, intention-to-treat analysis; At, as-treated analysis.

means of at least $30 \%$ and $10 \%$, respectively. For the MQS, a mean reduction of at least 4 in the crude score was considered a significant reduction.

The group differences in the VAS pain score and NDI were analyzed by using an ANCOVA adjusted for baseline values, age, sex, and employment status. Noninferiority of the IFSI to the TFSI was claimed when the mean and $95 \%$ CI of the outcome variables of the IFSI were at least equivalent to or worse by $<15 \%$ than the outcome variables of TFSI. ${ }^{15}$ The efficacy of IFSI and TFSI was also compared in terms of the MQS with a repeated measures ANOVA adjusted for age, sex, and employment status. Contrasts were used to test the presence of a linear tendency with time in each group. Statistical software used for analyses was SAS, Version 9.3 (SAS Institute, Cary, North Carolina).
(25\%), while the subjects receiving IFSI had a more even distribution with spondylosis at $50 \%$ compared with disk herniation at $43 \%$.

The mean level of worst pain felt during the intervention was similar in both the intention-to-treat and as-treated analyses respectively, at 7.1 and 7.3 for the TFSI group and 6.2 and 6.0 for the IFSI group. Similarly, the mean level of cervical pain reported at 30 minutes after the intervention was equivalent in both groups, at 3.1 in the intention-to-treat analysis and 3.2 in the as-treated analysis. No adverse events occurred following the interventions. At the 4-week follow-up, in the intention-to-treat analysis, 1 subject of the TFSI group reported having tinnitus and vertigo since the intervention and 1 subject in each group reported having headaches during the 2 days following the intervention. In the as-treated analysis, all the delayed adverse effects were reported in the TFSI group.

\section{Clinical Efficacy of IFSI and TFSI}

The clinical efficacy of IFSI and TFSI, in terms of VAS pain score reduction for a mean baseline VAS pain score of 62.4 and of NDI improvement, is presented in Table 2. Regarding the MQS outcome, neither type of intervention demonstrated a significant reduction in the medication-intake score for all time measurements.

We also performed a subgroup analysis of the etiologic imaging findings for IFSI and TFSI in terms of VAS pain score reduction to discern any difference between apparently more acute 
Table 3: Pain severity subgroup analysis of the etiologic imaging findings for intraarticular facet and transforaminal corticosteroid injections

\begin{tabular}{|c|c|c|c|c|}
\hline \multirow[b]{3}{*}{ Variable } & \multicolumn{4}{|c|}{ Subgroups } \\
\hline & \multicolumn{2}{|c|}{ Disk Herniation \pm Spondylosis } & \multicolumn{2}{|c|}{ Spondylosis } \\
\hline & IFSI & TFSI & IFSI & TFSI \\
\hline \multicolumn{5}{|l|}{ Subjects (No.) } \\
\hline It & 14 & 8 & 14 & 20 \\
\hline At & 13 & 9 & 16 & 18 \\
\hline \multicolumn{5}{|c|}{ VAS\% (mean) (95\% CI) } \\
\hline It & $65.0(31.2-98.9)$ & $8.0(+20.2-36.2)$ & $30.1(0.4-59.7)$ & $+1.6(+33.7-30.4)$ \\
\hline At & $46.5(9.4-83.6)$ & $22.7(+6.6-51.9)$ & $23.6(+6.7-53.9)$ & $5.6(+27.1-38.2)$ \\
\hline
\end{tabular}

Note:-VAS\% indicates the relative difference between postintervention and baseline VAS pain scores; It, intentionto-treat analysis; At, as-treated analysis.

\begin{tabular}{|c|c|c|}
\hline \multirow{2}{*}{$\begin{array}{l}\text { Variable and Baseline } \\
\text { VAS Pain Score } \\
(0-100)\end{array}$} & \multicolumn{2}{|r|}{ Groups } \\
\hline & IFSI & TFSI \\
\hline \multicolumn{3}{|l|}{ Subjects (No.) } \\
\hline It & 28 & 28 \\
\hline At & 29 & 27 \\
\hline \multicolumn{3}{|l|}{ VAS\% (mean) (95\% CI) } \\
\hline \multicolumn{3}{|l|}{20} \\
\hline It & $53.3(4.0-102.7)$ & $+83.4(+132.8$ to +34.0$)$ \\
\hline At & $41.6(17.1-100.3)$ & $+70.6(+128.5$ to +12.7$)$ \\
\hline \multicolumn{3}{|r|}{ 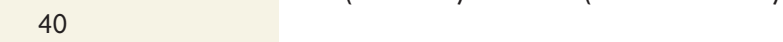 } \\
\hline It & $49.5(16.7-82.4)$ & $+39.4(+72.1$ to +6.6$)$ \\
\hline At & $39.4(0.6-78.2)$ & $+28.9(+66.9-9.2)$ \\
\hline \multicolumn{3}{|l|}{60} \\
\hline It & $45.8(21.8-69.8)$ & $4.6(+17.2-26.5)$ \\
\hline At & $37.2(9.4-65.0)$ & $12.9(+12.0-37.8)$ \\
\hline \multicolumn{3}{|l|}{80} \\
\hline It & $42.0(11.6-72.4)$ & $48.7(23.3-74.0)$ \\
\hline At & $35.0(+0.3-70.4)$ & $54.6(25.2-84.0)$ \\
\hline \multicolumn{3}{|l|}{95} \\
\hline It & $39.1(2.7-80.9)$ & $81.7(46.0-117.3)$ \\
\hline At & $33.4(15.6-82.3)$ & $86.0(44.1-127.8)$ \\
\hline
\end{tabular}

Note:-VAS\% indicates the relative difference between postintervention and baseline VAS pain scores; It, intention-to-treat analysis; At, as-treated analysis.

(disk herniation) and more chronic (spondylosis) causal factors. These results are presented in Table 3 .

\section{Analysis of Group Differences in Efficacy}

An interaction term was found between the randomized groups and the baseline VAS pain score $(P=.001)$. Hence, the efficacy of the interventions was linked to the level of the baseline VAS pain score (Table 4). The noninferiority analysis results for VAS pain scores are presented in Fig 3.

Regarding the NDI outcome, the difference in efficacy between IFSI and TFSI was $14.6 \%(95 \% \mathrm{CI},+18.4-47.7)$ in the intention-to-treat analysis and 7.9\% (95\% CI, +22.9-38.8) in the as-treated analysis. Although IFSI appeared to be more effective than TFSI at reducing the level of disability, this difference was not statistically significant and the result regarding noninferiority was inconclusive.

The difference in efficacy between the 2 groups in terms of the MQS score with time is presented in Fig 4. Although the visual perception would suggest a linear tendency for medication-intake reduction during the time in the IFSI group, this trend was not statistically significant both in the intention-to-treat analysis $(P=$
.654) and in the as-treated analysis $(P=$ .441). The hypothesis of a linear tendency for medication-intake reduction with time was also rejected for the TFSI group in both analyses $(P=.902, P=.675)$.

\section{DISCUSSION}

In 1997, Persson et $\mathrm{al}^{16}{ }^{16}$ in a randomized study, demonstrated that surgery, a custom physical therapy treatment, or the use of a cervical collar were equally effective at treating cervical radiculopathy. Because an inflammatory reaction is recognized as at least partly responsible for the irritation of the spinal nerve, corticosteroids should logically be part of the armamentarium used to treat this entity. Furthermore, to obtain optimal results, corticosteroids should be delivered at a high concentration as close as technically feasible to the site of the lesion. These principles provide the basis for the use of TFSI in the treatment of cervical radiculopathy. Notwithstanding that controlled studies demonstrating the efficacy of TFSI are lacking, the primary reason limiting the use of TFSI is the risk of serious complications. In an effort to determine the prevalence of severe complications, Scanlon et $\mathrm{al}^{7}$ performed a survey among the members of the American Spine Society. Among 1340 members, the response rate was $21.4 \%$ (287). In all, 78 complications were reported, including vertebrobasilar brain infarcts, cervical spinal cord infarcts, and 13 deaths. Arterial embolism of particulate corticosteroids is the most frequently cited presumptive cause of brain and spinal cord infarcts. ${ }^{17}$ While performing a TFSI, despite using a careful and precise technique, one can possibly cause inadvertent injection of material into radicular arteries that feed the spinal cord. ${ }^{18}$

The intention of this randomized controlled study was to test the hypothesis that IFSIs are at least as effective as TFSIs for the treatment of cervical radiculopathy. Both interventions were equally well tolerated by the subjects, and no major adverse events occurred with either type of intervention. For a mean baseline pain severity score, IFSI provided a clinically and statistically significant reduction in pain at 4 weeks' follow-up, while the improvement provided by TFSI was clinically and statistically nonsignificant. Furthermore, the subgroup analyses suggested that IFSIs were effective in subjects with cervical radiculopathy secondary to a disk herniation and maybe to a lesser degree in subjects with degenerative spondylosis, while TFSIs did not appear to provide significant relief in both subgroups. When we compared the efficacy of both interventions, an interaction with the baseline pain severity score was found. On the basis of these analyses, IFSIs were more effective or at least as effective as TFSIs for baseline pain severity scores of $\leq 60$, while whether TFSIs were more effective than IFSIs for baseline pain severity scores of $\geq 80$ could not be determined. Most interesting, for overall baseline pain severity scores between 20 and 95, IFSI provided significant relief for cervical radiculopathy. Conversely, TFSI appeared to be effective only in subjects presenting with baseline pain severity scores of $>80$. These last findings remain unclear. There was no relationship between the imaging findings and the baseline pain severity score, thus refuting the hypothesis that disk herniations could be 
associated with greater baseline pain severity than degenerative spondylosis and would respond better to TFSI.

Regarding the NDI outcome, while IFSI appeared to ease the

VAS\% Difference between IFSI and TFSI by VAS baseline value (intention to treat)

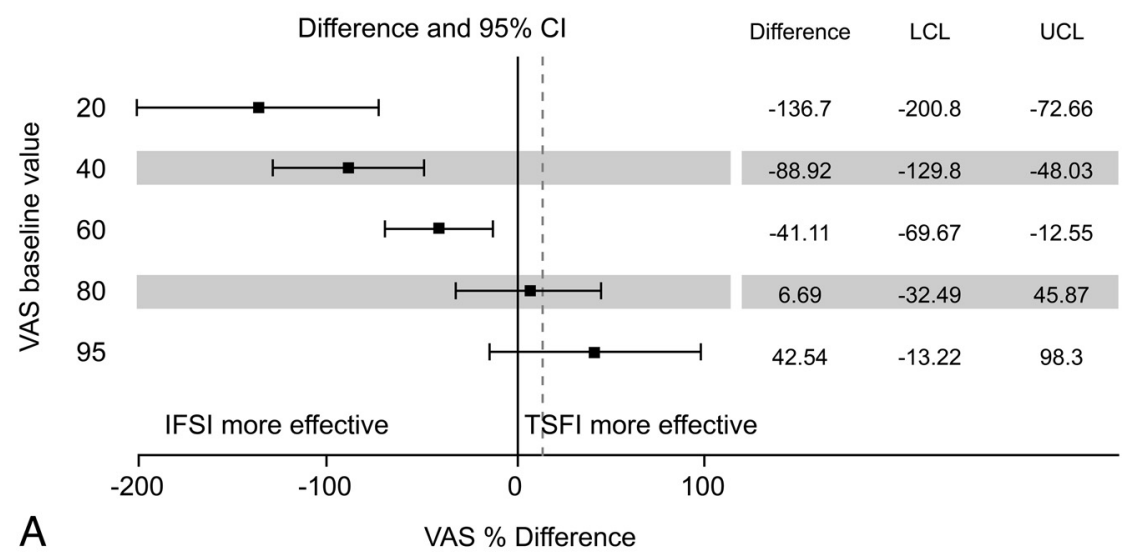

VAS $\%$ Difference between IFSI and TFSI by VAS baseline value (as treated)

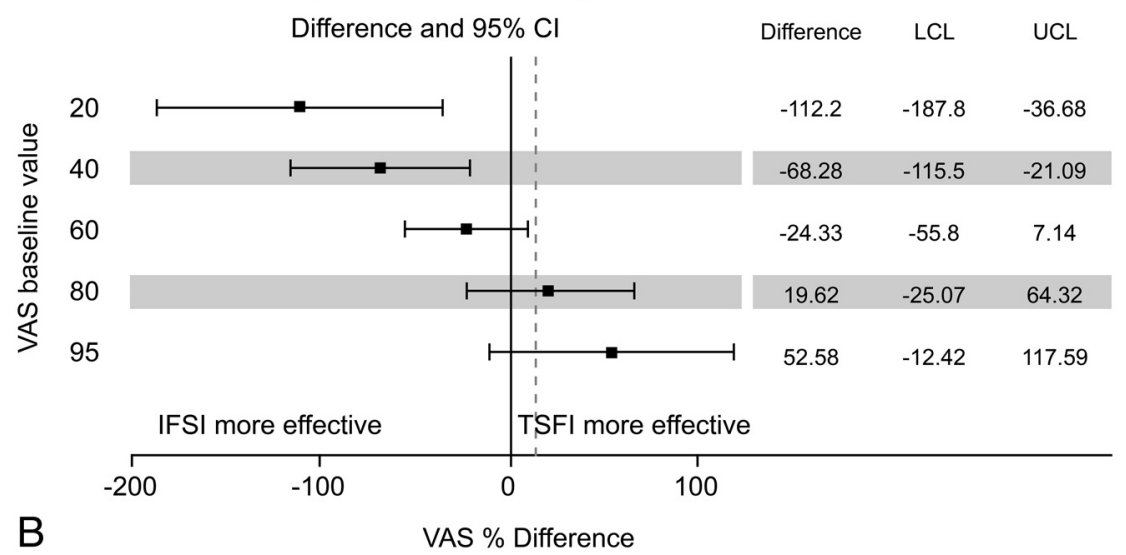

FIG 3. Relative Visual Analog Scale pain score differences between intra-articular facet and transforaminal corticosteroid injections, according to the VAS baseline value with adjustments for age, sex, and employment status. The intention-to-treat $(A)$ and as-treated $(B)$ analyses are presented. Error bars indicate 2-sided $95 \% \mathrm{Cls}$. Squares indicate mean differences. The dotted vertical line marks the $15 \%$ margin of noninferiority. LCL indicates lower confidence limit; UCL, upper confidence limit.

\section{Evolution of MQS score over time}

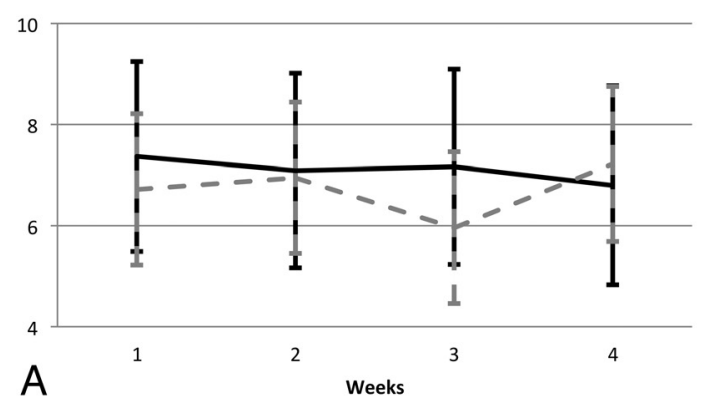

performance of specific daily activities on a clinical basis, the results did not reach statistical significance. When we compared the 2 interventions, IFSI appeared to be more effective than TFSI, but the difference was not statistically significant. Neither intervention was associated with a clinically significant pain medication-intake reduction with time.

An observational study by Kim et al in $2005^{12}$ suggested that IFSI could be effective in patients with cervical disk herniation. Unfortunately, the study group appeared to be inhomogeneous and the methodology was incompletely described for drawing any sound conclusions from that study. In 2007, Richarme et al ${ }^{19}$ presented preliminary results on contrast distribution following CT-guided intraarticular facet injections in 31 patients. Using this alternative approach, they obtained foraminal opacification in $21 / 31$ (68\%) patients and epidural opacification in $19 / 31$ (63\%). The following year, the same investigators presented their preliminary results on the efficacy of CTguided IFSI in 17 patients with cervical radiculopathy secondary to a disk herniation. ${ }^{11}$ They obtained pain relief of $>50 \%$ on a visual analog pain scale in $7 / 17$ (41\%) patients and reported extension of contrast in the foraminal space in $5 / 7$ patients with $>50 \%$ pain relief.

The exact mechanism of the apparent beneficial effect of IFSI for the treatment of cervical radiculopathy remains uncertain and unclear. As these studies suggest, 1 potential explanation is the proximity of the facet joint ventral capsular recess to the intervertebral foramen and/or leakage of the medication from the facet joint into the epidural and/or foraminal spaces. If this hypothetic mechanism of indirect delivery of corticosteroids to the site of pa-

FIG 4. Medication Quantitative Scale scores with time adjusted for age, sex, and employment status, for the intra-articular facet and transforaminal corticosteroid injections groups. The intention-to-treat $(A)$ and as-treated $(B)$ analyses are presented. Error bars indicate 2-sided $95 \%$ Cls. 
thology were the explanation for the efficacy of IFSI, then one would expect TFSI to provide at least equivalent results. In that regard, our results appear to be discordant with those of several noncontrolled, observational studies, which reported good-toexcellent results in anywhere from $24 \%$ to $76 \%$ of cases, ${ }^{5,6,20,21}$ including a more recent prospective case series study of 140 patients with chronic cervical radiculopathy due to degenerative spondylosis who received 3 consecutive TFSIs at 3-week intervals. ${ }^{22}$ These authors reported a significant pain reduction in $49 \%$ (69/140) of their patients at 12-14 weeks' follow-up.

Conversely, our results are in agreement with a randomized controlled study by Anderberg et al. ${ }^{23}$ These authors assigned 40 patients presenting with chronic cervical radiculopathy from degenerative spondylosis, with a mean duration of symptoms of 31 months, to receive 1 fluoroscopically guided TFSI. The treatment group received an injection of mepivacaine and methylprednisolone, while the control group received an injection of mepivacaine and saline. There was a positive response in only $30 \%$ $(6 / 20)$ of the patients in the treatment group at 3 weeks' followup. There were no significant differences in treatment results between the 2 groups.

The results of this study must be interpreted in light of some limitations. Facet joint syndrome may cause neck pain, which may radiate to the shoulders and can mimic cervical radiculopathy. ${ }^{24}$ We are confident that the randomized study design and the diagnosis of cervical radiculopathy made by specialists based on strict inclusion criteria with correlative imaging findings guarantee that the subjects in our study had cervical radiculopathy and that our results cannot be explained on the basis of a positive effect of IFSI on facet joint syndrome. While this randomized study allows evaluating the efficacy of IFSI and TFSI with a power of $80 \%$, our sample size is small for a study design of noninferiority comparing both interventions, to prove, without a doubt, that IFSIs are not less effective than TFSIs in treating cervical radiculopathy. This would require a prohibitively greater number of subjects. Nevertheless, this study is one of the few randomized controlled trials examining the efficacy of TFSI for the treatment of cervical radiculopathy and provides specialists who treat these patients and who perform these interventions with data to consider an alternative technique.

Although including 2 different etiologic causes for radiculopathy could be perceived as a limitation of this study, this is more representative of common clinical practice. According to Radhakrishnan et al, ${ }^{2}$ disk herniation is responsible for cervical radiculopathy in $20 \%-25 \%$ of cases, and spondylosis with or without disk herniation, in 70\%-75\% of the cases. Furthermore, regardless of the current etiology of cervical radiculopathy, the inclusion and exclusion criteria, the outcome measures, and the clinical question that we were addressing remained the same. Finally, although in our study we did not attempt to rupture the joint capsule while performing IFSI, we will address the issue of the potential interaction between contrast distribution and the efficacy of cervical corticosteroid injections in a future retrospective analysis of our data base.

\section{CONCLUSIONS}

IFSIs are effective for the treatment of cervical radiculopathy due to spondylosis and/or disk herniation. IFSIs were more or at least as effective as TFSIs in providing pain relief when the baseline pain severity score was low-to-moderate, while the comparison between the 2 interventions remained inconclusive for severe baseline pain level. IFSI can represent a valid and safer alternative to TFSI because no serious complications have been reported to date with this approach, to our knowledge. Consequently, we suggest that within an optimized medical treatment management, corticosteroid injections should initially be performed by using an intra-articular facet approach instead of a transforaminal approach.

\section{ACKNOWLEDGMENTS}

The authors thank Assia Belblidia for research assistance and Anne-Sophie Julien for statistical assistance.

Disclosures: Daniel Shedid—UNRELATED: Payment for Lectures (including service on Speakers Bureaus): Depuy Synthes, Fellowship Funds: Depuy Synthes and Medtronic.* *Money paid to the institution.

\section{REFERENCES}

1. Carette S, Fehlings MG. Clinical practice: cervical radiculopathy. N Engl J Med 2005;353:392-99

2. Radhakrishnan K, Litchy WJ, O'Fallon WM, et al. Epidemiology of cervical radiculopathy: a population-based study from Rochester, Minnesota, 1976 through 1990. Brain 1994;117(pt 2):325-35

3. Sampath P, Bendebba M, Davis JD, et al. Outcome in patients with cervical radiculopathy: prospective, multicenter study with independent clinical review. Spine 1999;24:591-97

4. Kang JD, Stefanovic-Racic M, McIntyre LA, et al. Toward a biochemical understanding of human intervertebral disc degeneration and herniation: contributions of nitric oxide, interleukins, prostaglandin E2, and matrix metalloproteinases. Spine 1997;22:1065-73

5. Cyteval C, Thomas E, Decoux E, et al. Cervical radiculopathy: open study on percutaneous periradicular foraminal steroid infiltration performed under CT control in 30 patients. AJNR Am J Neuroradiol 2004;25:441-45

6. Vallée JN, Feydy A, Carlier RY, et al. Chronic cervical radiculopathy: lateral-approach periradicular corticosteroid injection. Radiology 2001;218:886-92

7. Scanlon GC, Moeller-Bertram T, Romanowsky SM, et al. Cervical transforaminal epidural steroid injections: more dangerous than we think? Spine 2007;32:1249-56

8. Provenzano DA, Fanciullo G. Cervical transforaminal epidural steroid injections: should we be performing them? Reg Anesth Pain Med 2007;32:168, author reply 169-170

9. Wagner AL. CT fluoroscopic-guided cervical nerve root blocks. AJNR Am J Neuroradiol 2005;26:43-44

10. Hoang JK, Apostol MA, Kranz PG, et al. CT fluoroscopy-assisted cervical transforaminal steroid injection: tips, traps, and use of contrast material. AJR Am J Roentgenol 2010;195:888-94

11. Richarme D, Thevenin FS, Chevrot A, et al. Cervical radiculopathy: efficiency of CT-guided cervical facet joint corticosteroid injection. In: Proceedings of the 94th Annual Meeting of the Radiological Society of North America Annual Meeting. Chicago, Illinois; November 30December 5, 2008

12. Kim KH, Choi SH, Kim TK, et al. Cervical facet joint injections in the neck and shoulder pain. J Korean Med Sci 2005;20:659-62

13. Wlodyka-Demaille S, Poiraudeau S, Catanzariti JF, et al. French translation and validation of 3 functional disability scales for neck pain. Arch Phys Med Rehabil 2002;83:376-82

14. Harden RN, Weinland SR, Remble TA, et al. Medication Quantification Scale Version III: update in medication classes and revised detriment weights by survey of American Pain Society Physicians. J Pain 2005;6:364-71

15. Piaggio G, Elbourne DR, Altman DG, et al. Reporting of noninferi- 
ority and equivalence randomized trials: an extension of the CONSORT statement. JAMA 2006;295:1152-60

16. Persson LC, Carlsson CA, Carlsson JY. Long-lasting cervical radicular pain managed with surgery, physiotherapy, or a cervical collar: a prospective, randomized study. Spine 1997;22:751-58

17. Malhotra G, Abbasi A, Rhee M. Complications of transforaminal cervical epidural steroid injections. Spine (Phila Pa 1976) 2009; 34:731-39

18. Baker R, Dreyfuss P, Mercer S, et al. Cervical transforaminal injection of corticosteroids into a radicular artery: a possible mechanism for spinal cord injury. Pain 2003;103:211-15

19. Richarme D, Thevenin FS, Campagna R, et al. CT-guided cervical foraminal injection: is a direct foraminal approach still necessary? In: Proceedings of the 93rd Annual Meeting of the Radiological Society of North America. Chicago, Illinois; November 25-30, 2007

20. Bush K, Hillier S. Outcome of cervical radiculopathy treated with periradicular/epidural corticosteroid injections: a prospective study with independent clinical review. Eur Spine J 1996;5:31925

21. Lasbleiz J, Siegfried D, Chales G, et al. Evaluation of CT guided cervical epidural injections in patients with mechanical cervicobrachial neuralgia [in French]. J Radiol 2008;89(3 Pt 1):31723

22. Persson L, Anderberg L. Repetitive transforaminal steroid injections in cervical radiculopathy: a prospective outcome study including 140 patients. Evid Based Spine Care J 2012;3:1320

23. Anderberg L, Annertz M, Persson L, et al. Transforaminal steroid injections for the treatment of cervical radiculopathy: a prospective and randomised study. Eur Spine J 2007;16:321-28

24. Kirpalani D, Mitra R. Cervical facet joint dysfunction: a review. Arch Phys Med Rehabil 2008;89:770-74 